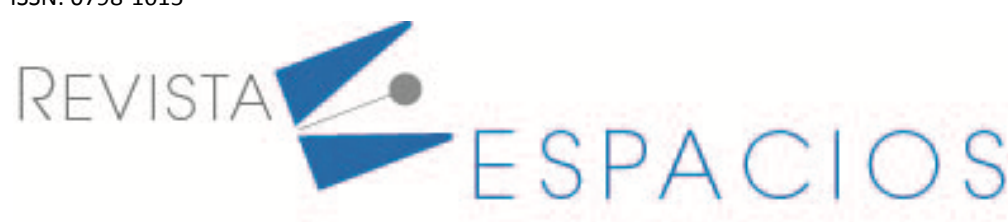

\title{
Análisis de los canales de YouTube como influencers del aprendizaje en Educación Primaria
}

\section{Analysis of YouTube channels as influencers of learning in Primary Education}

\author{
MARTÍNEZ-DOMINGO, José A. ${ }^{1}$ \\ TRUJILLO-TORRES, Juan M. ${ }^{2}$ \\ RODRÍGUEZ-JIMÉNEZ, Carmen ${ }^{3}$ \\ BERRAL-ORTIZ, Blanca ${ }^{4}$ \\ ROMERO-RODRÍGUEZ, José M. ${ }^{5}$
}

\begin{abstract}
Resumen
Las redes sociales y los Influencers se han hecho un hueco dentro de la enseñanza. Por consiguiente, el docente tiene que aprovechar estos recursos dentro del ámbito educativo. Por este motivo, se realiza un análisis de contenido de redes sociales de los canales de educación más influyentes de YouTube, observando diversas variables. Se llega a la conclusión de que es fundamental que un docente conozca las potencialidades de estos recursos para emplearlos dentro y fuera del aula.
\end{abstract}

Palabras clave: influencia del profesor, redes sociales, aulas virtuales, aprendizaje combinado

\begin{abstract}
Social networks and Influencers have made a place for themselves within teaching. Therefore, the teacher has to take advantage of these resources within the educational environment. For this reason, it is being done an content analysis of social networks of the most influential YouTube education channels, observing various variables. It is concluded that it is essential for a teacher to know the potential of these resources to use them inside and outside the classroom.

Key words: teacher influence, social networks, virtual classrooms, blended learning
\end{abstract}

\section{Introducción}

\subsection{Aprendizaje informal - internet}

Ha sido en las recientes décadas en las que la denominada era de la información y la comunicación ha conllevado intensos cambios en la vida de la sociedad. Estas transformaciones se deben a los cambios producidos en la organización del proceso productivo y también al surgir de un nuevo modelo sociotecnológico centrado en la microelectrónica, la información y el conocimiento (Burgos, 2017).

Con la llegada de las tecnologías e Internet, se produjo un cambio impactante en el modo de obtener la información. Esta no está concentrada en zonas exclusivas, ahora se puede acceder al conocimiento desde diversidad de lugares, en el momento que se desee y de modo ordenado, estando la información distribuida

\footnotetext{
${ }^{1}$ Becario de investigación. Departamento de Didáctica y Organización Escolar. Universidad de Granada, España. josemontejicar@correo.ugr.es

2 Personal Docente e Investigador. Departamento de Didáctica y Organización Escolar. Universidad de Granada, España. jttorres@ugr.es

${ }_{3}^{3}$ Personal Docente e Investigador. Departamento de Didáctica y Organización Escolar. Universidad de Granada, España. carmenrj@ugr.es

${ }^{4}$ Becaria de investigación. Departamento de Didáctica y Organización Escolar. Universidad de Granada, España. blancaberral@correo.ugr.es

${ }^{5}$ Personal Docente e Investigador. Departamento de Didáctica y Organización Escolar. Universidad de Granada, España. romejo@ugr.es
} 
(González-Sanmamed, Sangrá, Souto-Seijo y Estévez, 2018). El aprendizaje denominado como ubicuo, es decir, el que se logra en cualquier momento y en cualquier parte, hace pensar el modo en el que se produce el aprendizaje y lleva a hacer una reflexión sobre todas las aportaciones que generan las tecnologías de la información y la comunicación (Corpas, 2016; Wastiau et al., 2013).

En la actualidad, además de que los docentes innoven en el aula, deben de adquirir unas competencias y una actualización de conocimientos, para poder dar a la sociedad digital aquello que demanda (Hinojo, López, Fuentes, Pozo y Trujillo, 2020; Gudmundsdottir y Hatlevik, 2018).

Existen diferentes contextos en los que las personas intervienen, dentro de estos se integran la educación formal, la no formal y la informal (Tabuenca, Ternier y Specht, 2013; Pérez-Sanagustín, Alario-Hoyos y Kloos, 2014). Según Díaz (2016) es importante dentro de la educación diferenciar entre los tres tipos ya nombrados. En cuanto al tercer tipo, la educación informal, en el que más se incidirá en el presente trabajo, es definida como educación involuntaria, debido a que incide en la educación que adquiere la persona, sin tener un planteamiento previo. Esta puede adquirirse a través de medios de comunicación, pero también mediante el ejemplo que trasmiten los padres a sus hijos y la propia actitud del profesorado en clase.

El aprendizaje cotidiano y diario es el considerado como educación informal. Este se produce en sucesos o experiencias cotidianas, tal y como puede ser en el trabajo, con los compañeros o amigos y también en familia. Este tipo de aprendizaje no tiene una previa organización, es casual, esto se puede denominar como aprendizaje incidental (González-Sanmamed et al., 2018).

Internet es uno de los espacios en el que se produce aprendizaje informal a través de diversidad de procesos de aprendizaje online. El sistema educativo y la educación en general no lo puede dejar a un lado. Además, el hecho de que el alumnado introduzca en entornos de aprendizaje formal, estrategias y herramientas que permiten establecer aprendizaje informal, hace que sea muy significativo para el proceso de aprendizaje de los estudiantes (Pavani y Temporão, 2021).

\subsection{Ecologías del aprendizaje}

Actualmente, las distintas teorías del aprendizaje tienen que conocer de la existencia de diversos sistemas interconectados, complejos y a la vez ricos en contenidos. Aquí es donde se crea y comparte el conocimiento, resurgiendo el concepto de ecologías de aprendizaje (Tabuenca et al., 2013). Este término es una metáfora; por ecología se entiende tener una mirada mayor del aprendizaje, debido a que las formas en las que se produce aprendizaje son respetadas (Scolari, Masanet, Guerrero-Pico y Establés, 2018).

Por tanto, el concepto de ecología aporta la base para futuros modelos educativos que están más enlazados con el contexto que se presenta y, también, con las características del conocimiento más reciente, que es emergente, interdisciplinario y relativamente desordenado. Además, abarca diferentes interacciones, diversidad de contextos y procesos, los cuales aportan al sujeto distintos recursos que le permiten adquirir conocimientos, y también determinadas oportunidades, con el objetivo de desarrollar nuevas metas y aprendizajes (RomeuFontanillas, Guitert-Catasús, Raffaghelli y Sangrà, 2020).

Las ecologías de aprendizaje potencian las capacidades de análisis sobre cómo aprende una persona, qué elementos intervienen en esta formación y en los contextos dónde se produce, teniendo como objetivo presentar nuevas oportunidades de aprender. Gracias a ellas pueden interpretarse las diferentes oportunidades de aprendizaje que ofrece el presente panorama digital, donde los tres tipos de educación en ocasiones llevan a aprendizajes invisibles (González-Sanmamed et al., 2018). 


\subsection{Influencers del aprendizaje}

Las nuevas ecologías del aprendizaje, combinadas con la educación informal, hacen que se piense en un nuevo concepto, como es el de influencer centrado en el ámbito educativo. Un influencer es una persona que crea y transmite mediante sus redes sociales, un determinado contenido, además, tiene miles de seguidores. El contenido que emite suele ser de gran diversidad, y una vez que se sube a la red, este puede llegar a lugares muy lejanos, por lo que se puede decir que no tiene límites (Muiños, 2017; Ruiz, 2019).

Wei y Meng et al. (2021) afirman que los influencers son también personas que consumen y buscan abundante información y suelen hacerlo mucho más que la media de los demás individuos. Por tanto se puede establecer que los influencers son capaces de condicionar y transformar las opiniones de sus seguidores, no teniendo que ser personajes públicos destacados. Además, son individuos que producen un cambio en los demás mediante perfiles a través de los que influyen a muchas personas en las comunidades. Son agentes de cambio, perfiles que tienen una posición de poder para influir en las comunidades (Pérez, 2016; Khamis, Ang y Welling, 2017).

En relación al ámbito educativo, tal y como indican Romeu et al. (2020) existen docentes referentes, motivados por factores externos como son los históricos y contextuales, incidiendo estos en el proceso de EnseñanzaAprendizaje (EA). Estos factores hacen que los docentes se interesen por aumentar sus competencias y formación profesional, así como en el empleo de recursos digitales, y de realizar comunicaciones a través de medios online informales, entrando en contacto con las redes sociales. Estos docentes referentes se pueden entender como influyentes para el resto del profesorado que quiere actualizarse.

El profesorado que usa Tecnologías de linfrmación y Comunicación (TIC) tiende a buscar otros influyentes del aprendizaje, que les dote de nuevas ideas. Para ello, tienen que ser docentes que observen la relevancia de las innovaciones, ayudados por la curiosidad de aprender (Romeu et al., 2020).

A este respecto, Unal y Ozturk (2012) señalan que las búsquedas de docentes influyentes en las redes sociales se han desarrollado a gran velocidad en los años más recientes y han generado la posibilidad de crear nuevos ámbitos educativos, en el que se pueden destacar las redes sociales educativas, así como, avances significativos en cuanto a materiales y técnicas de EA.

\subsection{Redes sociales}

La entrada de los influencers en el ámbito educativo hace necesario destacar que, hoy en día, tal y como señala Scolari et al. (2018) todo el mundo está inmerso en una cultura digital y define el aprendizaje transmedia como las estrategias de aprendizaje y habilidades centradas en las nuevas comunidades virtuales, en las que se destacan nuevos conceptos e información que conocen los estudiantes más jóvenes.

A su vez, debido a los cambios que manifiesta el aprendizaje virtual, los docentes presentan interés por formarse en competencia digital, con el fin poder manejar las nuevas tecnologías. Esto, unido a los años de dedicación, se entienden como factores principales que inciden en que el profesorado que presenta una educación permanente, logre adquirir la competencia digital (Hinojo-Lucena, Aznar-Díaz, Cáceres-Reche, Trujillo-Torres y Romero-Rodríguez, 2019b).

Cabe destacar, que el docente además de ser competente digitalmente, tiene que conocer los riesgos que puede conllevar el uso de internet, como es la adicción a este. Cuando se usan de forma excesiva las nuevas tecnologías puede haber efectos negativos en la salud, tanto física como mental. Sin embargo, se pueden proponer medidas ante tales problemas, para conseguir evitarlos (Hinojo-Lucena, Aznar-Díaz, Cáceres-Reche, Trujillo-Torres y Romero-Rodríguez, 2019a). 
Es necesario que se analicen las distintas y enriquecedoras perspectivas de aprendizaje, centrándose en el propio estudiante, el cual presenta diferentes habilidades que le permiten aprender, así como distintas preferencias para utilizar las tecnologías que tiene cerca, transformando estas en posibilidades de aprender (Arenas, 2015). Una de estas posibilidades es a través de las redes sociales, aprovechándose plenamente los beneficios de las TIC (Dumpit y Fernández, 2017).

Las redessociaes son estructuras sociales que están integradas por personas que forman un grupo, al tener una misma relación, actividad o interés por medio de la red. Es en esta donde se producen diversidad de interacciones, donde coinciden individuos de forma virtual, pudiendo ser en tiempo real. También, se trasmiten las últimas innovaciones y modas, además de comentar opiniones de forma abierta, lo que caracteriza la identidad de la persona (Ponce, 2012; Kaplan y Haenlein, 2010).

En la actualidad, es una realidad que se han producido cambios en la estructura social, a causa de las redes sociales, y por consiguiente el modo en el que las personas se relacionan y comunican. Estas son ahora, espacios en los que se comparte el día a día, y en los que se produce un acceso rápido a la información y es una forma rápida de comunicarse (García, 2018).

Las redes sociales también aportan posibilidades pedagógicas y culturales, desde una perspectiva informal, destacándose entre otras Instagram, Facebook, Twitter, YouTube y WhatsApp. Esta última es habitual entre los estudiantes que intercambian apuntes, dudas o ánimos a través de este medio (Izquierdo-Iranzo y GallardoEchenique, 2020).

El presente estudio está basado en YouTube, una plataforma en la que, según Scolari (2018), se desarrollan las competencias transmedia de forma externa a los ámbitos de educación formal de aprendizaje. Además, en lo que respecta a la formación y al aprendizaje entre la juventud, los vídeos se entienden como recurso educativo y esto se refleja en que, de cada diez jóvenes españoles, ocho acceden a vídeos para formarse y aprender, es decir, el 96\% de los jóvenes de 14 a 19 años, y el 94,6\% en los que tienen de 20 a 24 años (Fundación Telefónica, 2017).

\subsection{Flipped classroom}

Tras todo lo comentado, se puede entender que el aprendizaje que se logra mediante las redes sociales, y a través de influencers, puede comprenderse como una metodología Flipped Classroom más vinculada al ámbito informal. Según Berenguer (2016) este método de enseñanza también conocido como aula invertida, aporta un papel más activo que el método tradicional en el proceso de aprendizaje. Por lo tanto, el alumnado con un papel activo, estudia los conocimientos que el docente facilita y la clase es dedicada a realizar debates que surjan del contenido visualizado, para realizar prácticas de lo aprendido y también, para resolver dudas (Wasserman, Quint, Norris y Carr, 2017; Abeysekera y Dawson, 2015; Gilboy, Heinerichs y Pazzaglia, 2015).

Cuando se utilizan vídeos en esta metodología de EA, se otorgan ventajas tales como permitir que el contenido de estos vídeos sea actualizado, pudiéndose visualizar las veces que sea necesario y ser usado en años posteriores, por lo que se caracteriza por ser un recurso educativo muy dinámico (Lara y Rivas, 2009).

\subsection{Objetivos}

El objetivo general del estudio es conocer los principales canales de educación de YouTube de España, que puedan servir tanto a docentes como alumnado desde el ámbito informal, o por parte del docente llevarlos al aula, pudiendo ser un recurso, que se envía para verse en casa como complemento del proceso de EA que se lleva a cabo, o también para aquellos alumnos que por ciertas necesidades o razones no pueden asistir a clase, por lo que se estaría introduciendo en la educación formal. 
Siguiendo las cuestiones presentadas por Aznar-Díaz et al. (2019) en su estudio, se plantean los siguientes interrogantes como eje de la investigación y se añade una última cuestión:

- ¿ ¿ ¿ ¿ caé canales españoles de YouTube de educación son los que presentan más influencia?

- $\quad$ ¿Cuál es el contenido de estos canales educativos de YouTube?

¿Tiene influencia en las visualizaciones y suscripciones, el número de vídeos subidos al canal?

¿Qué canales de educación de YouTube se relacionan entre sí debido al contenido que comparten?

¿Con qué otras redes sociales están vinculados los canales de YouTube?

\section{Metodología}

La metodología llevada a cabo es un Análisis de Redes Sociales, con el que se estudia la estructura social y, a su vez, también se utiliza para estudios de redes virtuales al centrarse en calcular indicadores de carácter cuantitativo, para analizar las relaciones entre personas o grupos, siendo el caso de modo virtual (Hawe, Webster y Shiell, 2004). En esta metodología es clave identificar variables que puedan relacionarse, analizándose la fuerza que presenta una vinculación y el tipo de relación, por lo que se identifican las principales relaciones (Lozares, 2005). Con el fin de conseguir responder el objetivo principal se emplea la técnica conocida como análisis de contenido (Mejía, 2010).

Para analizar cuál es la influencia que presentan los canales de YouTube, esta se consigue determinando el número de visualizaciones y suscriptores que presenta dicho canal (Pérez, Castro y Fandos, 2018). Mediante este método se puede, de forma objetiva y cuantitativa, analizar y estudiar el contenido de un canal (Hernández, Fernández y Baptista, 2016). Esta metodología destaca por haberse realizado en estudios anteriores relacionados con la red social YouTube (Aznar-Díaz et al., 2019; Montes, García y Menor, 2018; Tur-Viñes et al., 2018).

Por tanto, se plantea realizar un análisis de canales españoles de YouTube que versen sobre educación.

\subsection{Unidad de análisis}

Para seleccionar los canales educativos españoles de YouTube más influyentes, se han seguido las indicaciones de Pérez et al. (2018) que establece como criterio tener 100.000 suscriptores y 10.000 visitas como mínimo, para ser objeto de análisis. En esta investigación, la unidad de análisis final consta de un total de 16 elementos ( $\mathrm{N}=$ 16), los cuales son canales educativos españoles de YouTube más influyentes en el alumnado y docentes de Educación Primaria. En este estudio, la recopilación de datos tuvo lugar en 2020 durante la primera quincena de abril. Tal y como señalan Aznar-Díaz et al. (2019) se debe tener en cuenta que los canales de YouTube tienen gran dinamismo en lo que respecta a número de vídeos, visualizaciones y suscriptores.

Para este estudio, partiendo de los 16 canales de YouTube que se toman como unidad de análisis, se analizan las siguientes variables: año de creación del canal (V1), suscriptores del canal (V2), número de visualizaciones (V3) y vídeos totales (V4), estas cuatro se recogen en Frígola, Palomo y Marca (2015) y se añade otra variable, que se refleja en Aznar-Díaz et al. (2019) que es el tipo de contenido que presentan los vídeos (V5). Además, se analiza una última variable que se añade, que es conocer las redes sociales con las que se vincula cada canal (V6).

\subsection{Análisis de datos}

Tras la recopilación de los resultados, estos se han analizado con dos programas diferentes. Por un lado, con el programa estadístico IBM SPSS siendo la versión 25. Mediante el mismo se ha llevado a cabo una relación de variables con el fin de determinar la fiabilidad, con la correlación de Pearson, por lo que se ha realizado un análisis 
de regresión lineal entre el número de suscriptores (V2) y vídeos totales (V4) y, por otro lado, el mismo análisis entre el número de visualizaciones (V3) y la V4. Por otra parte, en lo que respecta a la variable V5, se han llevado a cabo conexiones entre los canales de YouTube para observar aquellos que más relación tienen. Este proceso se ha realizado con el programa de visualización de redes VOSviewer, en la versión 1.6.15. Por otro lado, con V5 se realiza una recopilación gráfica del contenido predominante en los 16 canales tomados como unidad final.

\section{Resultados}

Por un lado, se describen los 16 canales educativos de YouTube centrándose en las variables de la fecha de apertura (V1), el contenido (V5) y las redes sociales asociadas al canal (V6). El contenido indicado de cada canal, corresponde al que se relaciona con la etapa de Educación Primaria. Cabe destacar que los canales son recientes, en general se iniciaron a partir de 2013, siendo el más reciente de 2016. Por otro lugar, hay que destacar que también de forma general todos los canales de YouTube están vinculados a las redes sociales de Facebook, Twitter e Instagram, y tienen página web.

- Academia JAF: fecha de creación del canal 21 de agostos de 2013. El contenido se corresponde con las asignaturas de Matemáticas y Lengua Castellana y Literatura. Además, tiene otras redes sociales que son Facebook y Twitter, y página web.

- Academia Play: fecha de creación del canal 07 de septiembre de 2015. El contenido se corresponde con las asignaturas de Ciencias Sociales, Ciencias de la Naturaleza, Matemáticas, Educación Musical y Lengua Castellana y Literatura. Además, tiene otras redes sociales que son Facebook, Twitter e Instagram, y página web.

- Amigos Ingleses: fecha de creación del canal 23 de junio de 2012. El contenido se corresponde con la asignatura de Primera Lengua Extranjera (Inglés). Además, tiene otras redes sociales que son Facebook, Twitter e Instagram, y página web.

- Ciencias de la Ciencia: fecha de creación del canal 11 de enero de 2016. El contenido se corresponde con las asignaturas de Ciencias Sociales y Ciencias de la Naturaleza. Además, tiene otras redes sociales que son Facebook, Twitter e Instagram, y página web.

- El Mundo de Luna: fecha de creación del canal 26 de junio de 2015. El contenido se corresponde con las asignaturas de Educación Musical y Ciencias de la Naturaleza. Además, tiene otra red social que es Facebook.

- Eugenia Romero: fecha de creación del canal 19 de mayo de 2009. El contenido se corresponde con la asignatura de Lengua Castellana y Literatura. Además, tiene otras redes sociales que son Facebook, Twitter e Instagram, y página web.

- ExpCaserosKids: fecha de creación del canal 05 de septiembre de 2013. El contenido se corresponde con las asignaturas de Ciencias de la Naturaleza, Matemáticas y Primera Lengua Extranjera (Inglés). Además, tiene otras redes sociales que son Facebook, Twitter e Instagram, y página web.

- Happy Learning Español: fecha de creación del canal 27 de febrero de 2015. El contenido se corresponde con las asignaturas de Ciencias Sociales, Ciencias de la Naturaleza, Lengua Castellana y Literatura, Educación Musical, Matemáticas, Primera Lengua Extranjera (Inglés) y Educación Plástica. Además, tiene otras redes sociales que son Facebook, Twitter e Instagram, y página web. 
- Jaime Altozano: fecha de creación del canal 02 de junio de 2016. El contenido se corresponde con la asignatura de Educación Musical. Además, tiene otras redes sociales que son Facebook, Twitter e Instagram.

- La Eduteca: fecha de creación del canal 20 de febrero de 2012. El contenido se corresponde con las asignaturas de Ciencias Sociales, Ciencias de la Naturaleza, Lengua Castellana y Literatura, y Matemáticas. Además, tiene otras redes sociales que son Facebook, Twitter e Instagram, y página web.

- LlegaExperimentos: fecha de creación del canal 16 de mayo de 2013. El contenido se corresponde con las asignaturas de Ciencias de la Naturaleza. Además, tiene otras redes sociales que son Facebook, Twitter e Instagram, y página web.

- $\quad$ NIJI: fecha de creación del canal 24 de marzo de 2010. El contenido se corresponde con las asignaturas de Primera Lengua Extranjera (Inglés), Matemáticas y Lengua Castellana y Literatura. Este canal no tiene otras redes sociales ni página web.

- Podemos aprobar matemáticas: fecha de creación del canal 17 de marzo de 2013. El contenido se corresponde con la asignatura de Matemáticas. Además, tiene otras redes sociales que son Facebook y Twitter.

- Smile and Learn - Español: fecha de creación del canal 15 de noviembre de 2016. El contenido se corresponde con las asignaturas de Ciencias Sociales, Ciencias de la Naturaleza, Matemáticas, Lengua Castellana y Literatura, Primera Lengua Extranjera (Inglés), Educación Musical y Educación Plástica. Además, tiene página web y aplicación informática.

- unProfesor: fecha de creación del canal 16 de diciembre de 2013. El contenido se corresponde con las asignaturas de Matemáticas, Lengua Castellana y Literatura, Ciencias Sociales, Ciencias de la Naturaleza y Educación Musical. Además, tiene otras redes sociales que son Facebook y Twitter, y página web.

- YouTalk TV: fecha de creación del canal 18 de febrero de 2013. El contenido se corresponde con la asignatura de Primera Lengua Extranjera (Inglés). Además, tiene otra red social que es Instagram.

En lo que respecta al número de suscriptores, visualizaciones y vídeos, V2, V3 y V4, respectivamente, se presentan los resultados de dichas variables en la Tabla 1. Los canales en total muestran que la media de vídeos es 614, la de número de suscriptores es 970.875 y de número de visualizaciones 135.532.918.

Por otro lado, en lo referente al análisis de regresión lineal, al relacionar la variable del número de vídeos (V4) y suscriptores (V2) ( $r=-0,177$, y $p=0,512)$, por lo que el coeficiente de correlación indica que es negativa y no significativa tal y como se muestra en la Figura 1. Al relacionar la V4 y el número de visualizaciones (V3) ( $r=-$ 0,136 , y $p=0,616$ ) por lo que la correlación es negativa y no significativa, mostrándose en la Figura 2 . Esto se podría entender como que a mayor número de vídeos no se producirá un aumento ni de visualizaciones ni de suscriptores, por lo que entrarían en juego el factor del contenido y el modo en que se explica el mismo, las redes sociales a las que se asocia el canal y, también, al factor económico, es decir, lo que se invierte para promocionar el canal (Aznar-Díaz et al., 2019). 
Tabla 1

Datos sobre el número de vídeos, suscriptores y visualizaciones de cada canal

\begin{tabular}{lccc}
\hline \multicolumn{1}{c}{ Canal } & No de vídeos & No de suscriptores & No de visualizaciones \\
\hline academia JAF & 452 & 112.000 & 16.694 .749 \\
Academia Play & 248 & 2.010 .000 & 141.188 .047 \\
Amigos Ingleses & 268 & 1.080 .000 & 54.013 .236 \\
Ciencias de la Ciencia & 184 & 143.000 & 4.260 .734 \\
El Mundo de Luna & 73 & 363.000 & 123.364 .336 \\
Eugenia Romero & 143 & 237.000 & 150.601 .799 \\
ExpCaserosKids & 315 & 3.270 .000 & 547.019 .477 \\
Happy Learning Español & 330 & 834.000 & 168.618 .201 \\
Jaime Altozano & 166 & 2.250 .000 & 154.381 .167 \\
La Eduteca & 193 & 169.000 & 44.958 .634 \\
LlegaExperimentos & 376 & 2.610 .000 & 366.328 .442 \\
NIJI & 395 & 114.000 & 57.001 .297 \\
Podemos aprobar & & & \\
matemáticas & 3.899 & 294.000 & 62.778 .206 \\
Smile and Learn - & 490 & 522.000 & 115.887 .080 \\
Español & 1.677 & 931.000 & 139.142 .685 \\
unProfesor & 615 & 595.000 & 22.288 .598 \\
YouTalk TV & & &
\end{tabular}

En lo que respecta a las regresiones lineales realizadas, en ambos casos se explica que hay un $3 \%$ y un $1 \%$ de variabilidad del eje $Y$, basándose en el promedio $\left(R^{2}=0,03\right.$ (Figura 1); $R^{2}=0,01$ (Figura 2 ).

Figura 1

Relación entre número de vídeos y suscriptores

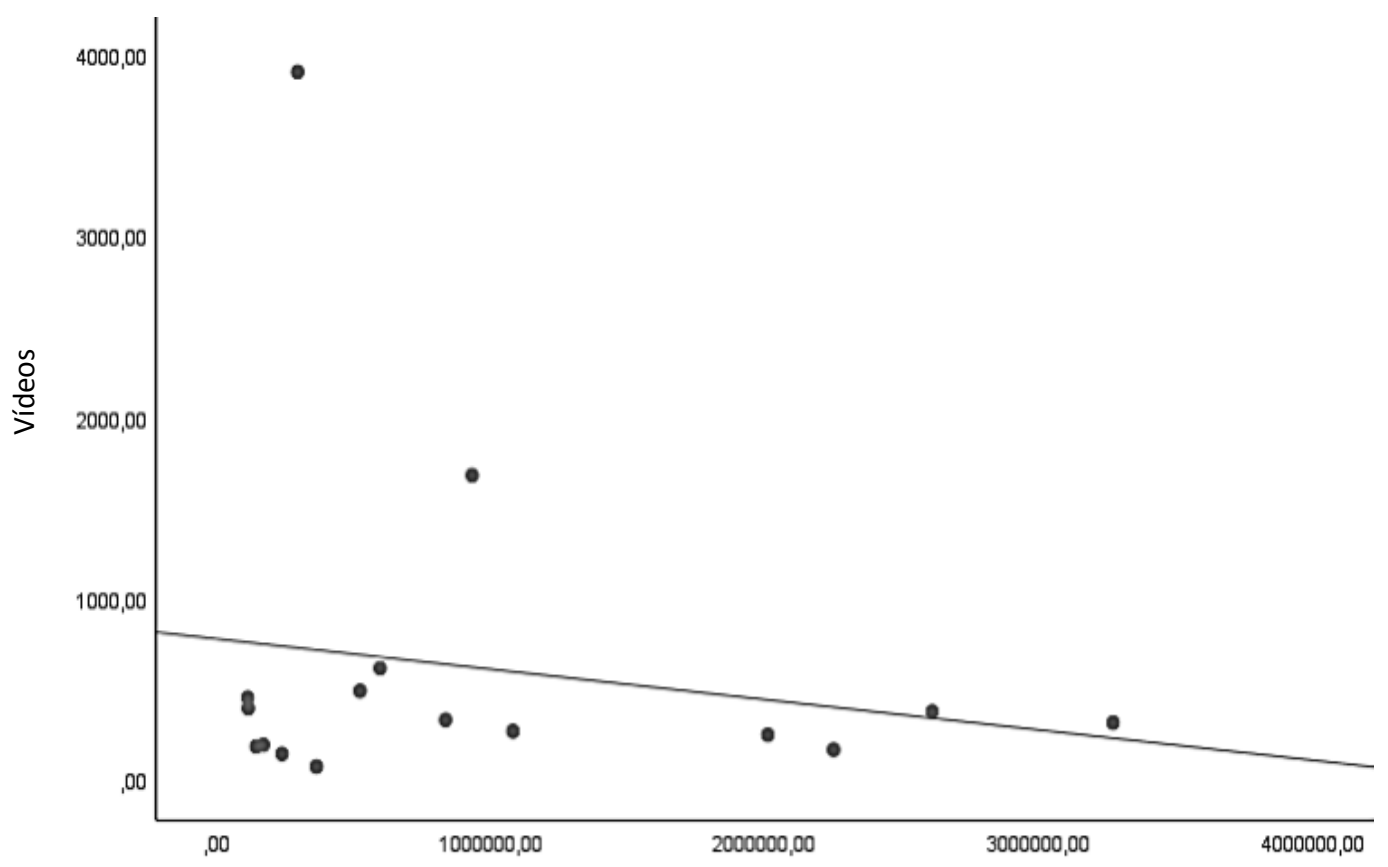

Fuente: Elaboración propia 
Figura 2

Relación entre número de vídeos y visualizaciones

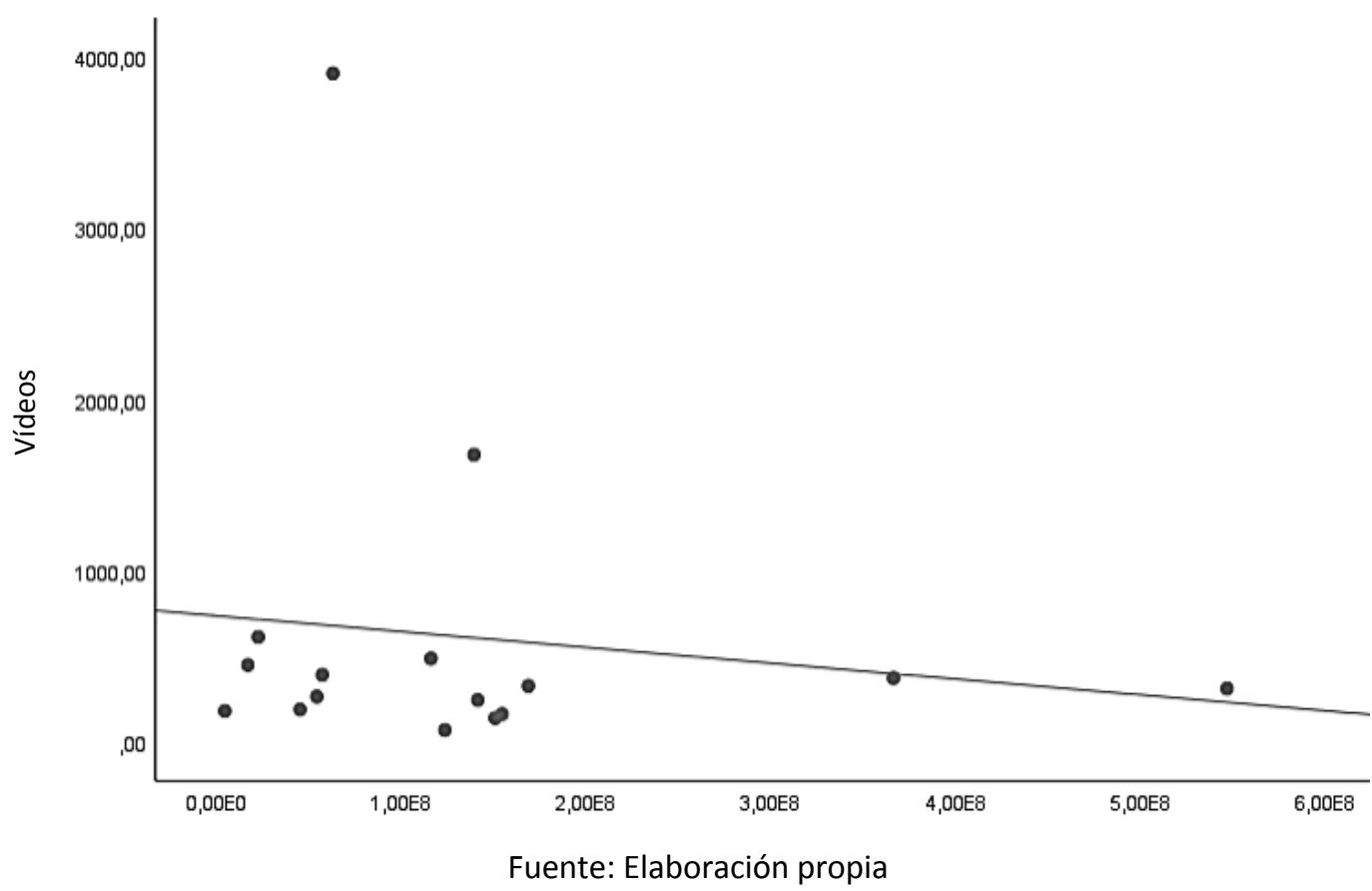

Por otra parte, se hizo un análisis del contenido de forma detallada de cada canal de YouTube. Se debe matizar que el contenido de estos canales se clasifica en asignaturas educativas, las presentes en estos canales de forma general y de mayor a menor presencia en los mismos son: Matemáticas, Ciencia de la Naturaleza, Lengua Castellana y Literatura, Primera Lengua Extranjera (Inglés), Ciencias Sociales, Educación Musical y Educación Plástica. De los 16 canales tomados como unidad final, las asignaturas que tienen mayor presencia son Matemáticas y Ciencias de la Naturaleza, siendo contenido de 9 de los 16 canales educativos, tal y como se muestra en el Gráfico 1.

\section{Gráfico 1}

Asignaturas predominantes en los canales educativos de YouTube 
Por otro lado, se lleva a cabo la relación entre canales de YouTube para identificar los canales que están más relacionados por su contenido. Como se ha mencionado este contenido se divide en asignaturas, y las que están más presentes son: Matemáticas (56\%), Ciencias de la Naturaleza (56\%) y Lengua Castellana y Literatura (50\%). Entonces, para establecer dicha relación se ha diseñado un mapa de nodos que destaca la conexión entre canales, cada círculo presenta un tamaño, el cual muestra mayor número de relaciones con los demás canales, al ser de mayor tamaño y, también se indica un color, el cual determina la cantidad de relaciones, de esta forma se indica en la Figura 3. Por consiguiente, tal y como señala Aznar-Díaz et al. (2019) se obtienen tres clústeres. El primero, destaca por el color rojo (Happy Learning-Español, Smile and Learn-Español, NIJI, ExpCaserosKids, Amigos Ingleses y YouTalk TV). En segundo lugar, el clúster de color azul (La Eduteca, Academia JAF, Podemos aprobar matemáticas y Eugenia Romero). El tercer clúster verde (Academia Play, unProfesor, Ciencias de la Ciencia, El Mundo de Luna, LlegaExperimentos y Jaime Altozano).

Figura 3

Relación entre los canales educativos españoles de YouTube

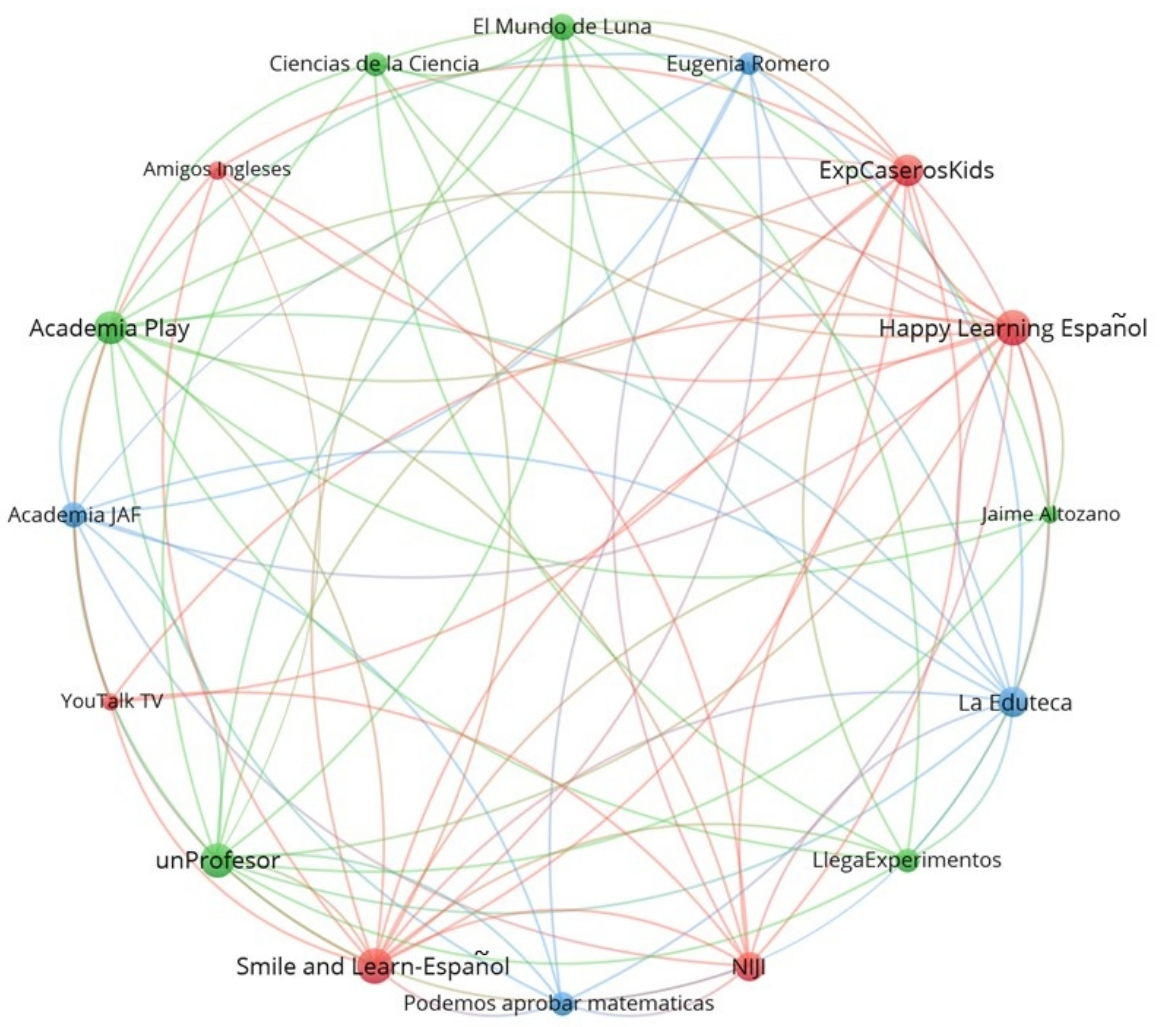

Fuente: Elaboración propia

\subsection{Discusión de resultados}

En la presente investigación, los resultados obtenidos muestran que los canales de YouTube tienen una gran influencia, específicamente los educativos, al poder comprobar que existen canales educativos con miles de suscriptores y millones de visualizaciones, que están destinados a la población de la etapa de Educación Primaria, y que partiendo de estos datos son muy influyentes para alumnos y docentes, por lo que también influyen en el aprendizaje, y es un hecho de actualidad debido a que, de forma general, todos los canales tienen una fecha de apertura dentro de esta última década. Esto se contrasta con lo que afirma Rodríguez y Fernández (2017) que YouTube está mostrando una mayor influencia en el proceso de EA, y que va aumentando. Además, en la actualidad se utilizan vídeos como recurso educativo como parte del entorno personal de aprendizaje (Lima, 
Rangel, Guimarães, y Marcelino, 2016; DelSignore, Wolbrink, Zurakowski y Burns, 2016). Cabe destacar que los alumnos que son nativos digitales, son una gran parte de los seguidores de los canales de YouTube, al ser gratuitos y no tienen que ser descargados, algo que facilita también el aprendizaje a través de vídeos (AlcaláSantaella, 2015).

En relación al Análisis de Redes Sociales tomado como metodología de investigación, para entender la interacción entre personas a través de internet, se entiende como algo nuevo en la investigación científica. Esta metodología permite usar sistemas visuales y matemáticos para facilitar el aprendizaje del proceso. Además, aporta información del proceso llevado a cabo por los individuos que constituyen una red, y la influencia que tiene (Torres, Álvarez, Gutiérrez y Ávila, 2012). Una vez conocida lo novedosa que esta metodología, los estudios con los que contrastar información son escasos.

El estudio aportado por Aznar-Díaz et al. (2019) es el más relacionado con la presente investigación llevada a cabo. Al contrastar información con dicho estudio se observa que en lo que respecta a las variables dos, tres y cuatro, número de suscriptores, visualizaciones y vídeos respectivamente, que se analiza realizando una regresión lineal de variables, se observa la relación que hay entre el número de vídeos y de suscriptores $(r=0,26$; $p=0,33)$, por lo que es positiva pero no significativa. En cuanto al número de vídeos y de visualizaciones $(r=$ 0,$48 ; p=0,06$ ) la relación es positiva pero no significativa. Sin embargo, en la investigación que se lleva a cabo, al relacionar la variable del número de vídeos (V4) y suscriptores (V2) ( $r=-0,177$, y $p=0,512)$, el coeficiente de correlación indica que es negativa y no significativa. Al relacionar la V4 y el número de visualizaciones (V3) ( $r=-$ 0,136 , y $p=0,616$ ) también la relación es negativa y no significativa.

Debido a lo comentado, en el primer estudio que versa sobre niños que son youtubers, en este caso no influye el número de vídeos del canal en los suscriptores ni visualizaciones del mismo, por tanto, el resultado coincide con la presente investigación, por lo que se entiende que tiene que haber otras variables que repercutan, como podrían ser el contenido y las redes sociales a las que un canal de YouTube está asociado, además de las inversiones económicas que apuestan por un canal.

En cuanto al contenido (V5), las asignaturas que predominan son Matemáticas y Ciencias de la Naturaleza, y esto se puede deber a las dificultades que presenta el alumnado en dichas materias, y en la comprensión de ciertos contenidos. En este sentido, Chamorro y Barletta (2009) afirman que, en sus investigaciones sobre la asignatura de Ciencias de la Naturaleza, existe información incompleta, referencias que no son correctas y, además, observan que no se establecen relaciones concretas entre lo escrito, los términos y el lenguaje ambiguo, y a esto se le añade que hay docentes que no son conscientes de estas dificultades, por lo que, se agravan al no tener un correcto seguimiento efectivo por el docente. Esto hace que, tal y como señala Colectivo Urdimbre (2000), el alumnado alcance dos tipos de aprendizaje, el recitado y el mecánico, produciéndose una elaboración incorrecta de la información.

Por otro lado, en lo que respecta a la asignatura de Matemáticas Siegenthaler, Miranda, Mercader y Presentación (2017) afirman que, siguiendo estudios en educación y psicología, existen ciertas competencias matemáticas que son básicas en las que se presenta dificultad, tal y como es el conteo y las dificultades en los procedimientos del mismo (Geary y Hoard, 2005; Ostad, 2000) y también la comparación de magnitudes y las operaciones lógicas, siendo estas competencias esenciales para el aprendizaje de las matemáticas.

En lo que respecta a si un canal tiene otras redes sociales (V6) y su influencia, dejando a un lado la variable del contenido, según Alcalá-Santaella (2015) YouTube se considera una red social entendida como un gran adelanto que da la posibilidad de difundir infinidad de contenidos por medio del soporte audiovisual. Esto permite a cualquier persona, generar contenido y difundirlo, además, si este contenido se comparte por medio de otras redes sociales, el impacto que tiene es mayor y el éxito del canal de YouTube es mucho mayor. 
Por lo tanto, podría entenderse que un canal educativo es más seguido cuando también está asociado a otras redes sociales, y este es el caso en la presente investigación, en la que se ha comprobado que no depende del número de vídeos, sino que podría ser del contenido y de las redes sociales en las que este se difunde.

\section{Conclusiones}

Los resultados de esta investigación educativa reflejan el logro del objetivo general que reflejaba el conocimiento de los principales canales españoles de educación de YouTube, con el objetivo de que sirvieran a los docentes y al alumnado en el proceso de EA, como recurso educativo. Después de realizar un análisis de numerosos canales de YouTube, se pudieron hallar los más influyentes en educación que son los siguientes: academia JAF, Academia Play, Amigos Ingleses, Ciencias de la Ciencia, El Mundo de Luna, Eugenia Romero, ExpCaserosKids, Happy Learning Español, Jaime Altozano, La Eduteca, LlegaExperimentos, NIJI, Podemos aprobar matemáticas, Smile and Learn-Español, YouTalk TV y unProfesor.

En cuanto al contenido, cabe decir que la asignatura de Educación Física no aparece como contenido de ningún canal de los destacados como más influyentes, pero sí se encontraron canales con este contenido, pero no alcanzaban el límite de 100.000 suscriptores.

Por otro lado, se ha analizado si tiene o no influencia en las visualizaciones y suscripciones, el número de vídeos subidos al canal y se ha podido comprobar que no tiene influencia, a mayor número de vídeos, los canales de educación no tienen más suscriptores ni visualizaciones, además en otra investigación como se ha relacionado anteriormente estas variables no tenían relación tampoco, por lo que se podría concluir que es el contenido y las redes sociales, a las que un canal está vinculado, lo que hace que un canal tenga más impacto.

Por otro lugar, se ha tratado de analizar qué otras redes sociales están vinculadas a los canales de YouTube de educación. Y se ha obtenido como resultado que las tres principales redes sociales son Facebook, Twitter e Instagram, en este mismo orden, además de tener página web.

Además de YouTube, se analizaron otras redes sociales asociadas a los canales de YouTube, tales como Facebook e Instagram, y como regla general muestran recursos educativos complementarios a los vídeos que suben al canal, y en algunos casos fragmentos de los vídeos, pero se toma el canal como principal vía de aporte educativo. Las redes sociales, como se ha comprobado anteriormente, aportan mayor propaganda al canal, lo que puede conllevar un mayor número de suscriptores y visualizaciones.

En relación a la prospectiva de este estudio se puede plantear, analizar de una forma más profunda el contenido de los canales educativos de YouTube y cómo el contenido se presenta en cada asignatura y canal, así como la duración media de los vídeos de los canales, para comprobar si esta puede ser una variable que afecte a la gran influencia que estos presentan. Además, se puede investigar cuál es la influencia real que tienen las redes sociales en los canales de YouTube asociados.

Esta investigación presenta limitaciones, una de ellas es que algunos no son canales exclusivos para alumnos de Educación Primaria, también dentro de estos hay apartados destinados a alumnado de Educación Infantil y de Secundaria, por lo que también puede influir en los resultados.

Otra limitación es que se centra en que un canal tenga más de 10.000 visualizaciones y 100.000 suscriptores, y aunque se tiene presente la calidad del contenido, no se analiza de forma profunda, aunque esto también puede ser clave para la influencia de estos canales. Analizando otros canales con menos suscriptores, se ha observado que sus explicaciones eran buenas y de calidad, pero no llegaban al número de suscriptores.

Así pues, los canales de YouTube educativo son un recurso que puede ser utilizado por docentes para completar sus clases o incluso para explicar un contenido y, además, es óptimo para el alumnado, para poder comprender 
un contenido que se resiste a entender, pudiendo ser visualizado varias veces e incluso explicado por varios docentes. Además, en una situación en la que el alumnado no pueda asistir al aula, por cualquier motivo, puede ser una herramienta eficaz para solventar las necesidades educativas que se planteen.

\section{Referencias bibliográficas}

Abeysekera, L., y Dawson, P. (2015). Motivation and cognitive load in the flipped classroom: definition, rationale and a call for research. Higher Education Research \& Development, 34(1), 1-14. doi: 10.1080/07294360.2014.934336

Alcalá-Santaella, M. (2015). De los escenarios presenciales a los virtuales: YouTube y el público juvenil. En J.M. Rodríguez (Ed.), Repensar los valores clásicos del periodismo: el desafío de una profesión enred@da: actas de las comunicaciones presentadas en el congreso (pp. 413-421). Madrid: Sociedad Española de Periodística. Recuperado de https://dialnet.unirioja.es/servlet/articulo?codigo=7293040

Arenas, E. (2015). Affordances of Learning Technologies in Higher Eduction Multicultural Environments. The Electronic Journal of e-Learning, 13(4), 217-227. Recuperado de https://eric.ed.gov/?id=EJ1062119

Aznar-Díaz, I, Trujillo-Torres, J. M., Romero-Rodríguez, J. M., y Campos-Soto, M. N. (2019). Generación Niños YouTubers: análisis de los canales YouTube de los nuevos fenómenos infantiles. Pixel-Bit. Revista de Medios y Educación, 56, 113-128. doi: 10.12795/pixelbit.2019.i56.06

Berenguer, C. (2016). Acerca de la utilidad del aula invertida o flipped classroom. En M. Tortosa, S. Grau, y J. Álvarez (eds.), XIV Jornadas de redes de investigación en docencia universitaria. Investigación, innovación y enseñanza universitaria: enfoques pluridisciplinares (pp. 1466- 1480). Universitat d'Alacant, Alicante. Recuperado de http://hdl.handle.net/10045/59358

Burgos, E. (2017). El aprendizaje informal y las habilidades transmedia. Temas de comunicación, (34), $26-41$. Recuperado de https://dialnet.unirioja.es/servlet/articulo?codigo=7283081

Chamorro, D., y Barletta, N. (2009). El lenguaje del texto escolar de Ciencias Naturales: problemas para el aprendizaje. Bio-grafía: escritos sobre la biología y su enseñanza, 2(2), 16-36. doi: 10.17227/20271034.vol.2num.2bio-grafia16.36

Colectivo Urdimbre. (2000). Libros de texto y aprendizaje en la escuela. Sevilla: Díada.

Corpas, M. D. (2016). La educación informal en el aprendizaje del inglés como lengua extranjera. Tejuelo: Didáctica de la Lengua y la Literatura. Educación, (24), 68-91. doi: 10.17398/1988-8430.24.1.68

DelSignore, L., Wolbrink, T., Zurakowski, D., y Burns, J. (2016). Test-enhanced e-learning strategies in Postgraduate Medical Education: A randomized cohort study. Journal of Medical Internet Research, 18(11). doi: https://doi.org/10.2196/jmir.6199

Díaz, M. (2016). Aprendizaje informal online. Mosaico. Revista para la promoción y apoyo a la enseñanza del español, (34), 56-61. Recuperado de https://sede.educacion.gob.es/publiventa/mosaico-n-34-revistapara-la-promocion-y-apoyo-a-la-ensenanza-del-espanol/ensenanza-lengua-espanola/21828

Dumpit, D. Z., y Fernandez, C. J. (2017). Analysis of the use of social media in Higher Education Institutions (HEls) using the Technology Acceptance Model. International Journal of Educational Technology in Higher Education, 14(5), 1-16. doi: 10.1186/s41239-017-0045-2 
Frígola, J., Palomo, A. M., y Marca, G. (2015). Análisis de los canales vídeos de las asociaciones médicas españolas. Opción, 31(6), 305-317. Recuperado de https://dialnet.unirioja.es/servlet/articulo?codigo $=5758718$

Fundación Telefónica (2017). Sociedad digital en España, 2017. España: Ariel. Recuperado de https://bit.ly/2IOc7RH

Garcia , H. (2018). ¿Están los influencers perdiendo su credibilidad?: Estudio sobre la credibilidad que el consumidor otorga al mensaje emitido por el influencer en la plataforma Instagram dentro del sector cosmético (Trabajo de Fin de Máster). Universidad Autónoma de Barcelona, Barcelona. Recuperado de https://ddd.uab.cat/record/200615

Geary, D. C. y Hoard, M. K. (2005). Learning disabilities in arithmetic and mathematics. En J.K. Campbell (ed.), Handbook of Mathematical Cognition (pp. 253-268). New York: Psychology Press. Recuperado de https://citeseerx.ist.psu.edu/viewdoc/download?doi=10.1.1.380.644\&rep=rep1\&type=pdf

Gilboy, M. B., Heinerichs, S., y Pazzaglia, G. (2015). Enhancing student engagement using the flipped classroom. Journal of nutrition education and behavior, 47(1), 109-114. doi: 10.1016/j.jneb.2014.08.008

González-Sanmamed, M., Sangrá , A., Souto-Seijo, A., y Estévez, I.E. (2018). Learning ecologies in the digital age: Challenges for higher. Publicaciones de la Facultad de Educación y Humanidades del Campus de Melilla, 48(1), 11-38. doi:10.30827/publicaciones.v48i1.7329

Gudmundsdottir, G. B., y Hatlevik, O. E. (2018). Newly qualified teachers' professional digital competence: implications for teacher education. European Journal of Teacher Education, 41(2), 214-231. doi: 10.1080/02619768.2017.1416085

Hawe, P., Webster, C., y Shiell, A. (2004). A glossary of terms for navigating the field of social network analysis. Journal Epidemiology Community Health, 58(12), 971-975. doi: 10.1136 / jech.2003.014530

Hernández, R., Fernández, C., y Baptista, P. (2016). Metodología de la investigación. México: McGraw-HillInteramericana de México.

Hinojo, F. J., López, J., Fuentes, A., Pozo, S., y Trujillo, J. M. (2020). Academic effects of the use of flipped learning in Physical Education. International Journal of Environmental Research and Public Health, 17(1), 276.

doi: 10.3390/ijerph17010276

Hinojo-Lucena, F. J., Aznar-Díaz, I., Cáceres-Reche, M. P., Trujillo-Torres, J. M., y Romero-Rodríguez, J. M. (2019a). Factors influencing the development of digital competence in teachers: Analysis of the teaching staff of Permanent Education centres. IEEE Access, 7(1), 178744-178752. doi:

10.1109/ACCESS.2019.2957438

Hinojo-Lucena, F. J., Aznar-Díaz, I., Cáceres-Reche, M. P., Trujillo-Torres, J. M., y Romero-Rodríguez, J. M. (2019b). Problematic Internet use as a predictor of eating disorders in students. A systematic review and meta-analysis study. Nutrients, 11(9), 2151. doi: 10.3390/nu11092151

Izquierdo-Iranzo, P., y Gallardo-Echenique, E. (2020). Studygrammers: Learning influencers. Comunicar, 28(62), 115-125. doi: 10.3916/C62-2020-10

Kaplan, A. M., y Haenlein, M. (2010). Users of the world, unite! The challenges and opportunities of Social Media. Business horizons, 53(1), 59-68. doi: 10.1016/j.bushor.2009.09.003 
Khamis, S., Ang, L., y Welling, R. (2017). Auto-branding, 'micro-celebridades' y el auge de los Influencers de las Redes Sociales. Estudios de celebridades, 8(2), 191-208. doi: 10.1080/19392397.2016.1218292

Lara, S., y Rivas, S. (2009). Aprendizaje autorregulado y fomento de competencias en dos asignaturas de Master a través del empleo de plantillas de evaluación, método del caso, role-playing y vídeo digital. Educación XX1, 12, 67-96. doi: 10.5944/educxx1.1.12.288

Lima, R., Rangel, F., Guimarães, M., y Marcelino, V. (2016). Diabetes mellitus: Generating issues for the teaching of biochemistry. Journal of Biochemistry Education, 14(2), 15. doi: 10.16923/reb.v14i2.668

Lozares, C. (2005). Valores, campos y capitales sociales. REDES. Revista Hispana para el Análisis de Redes Sociales, 4(2). doi: 10.5565/rev/redes.41

Mejía, C. (2010). Análisis de Redes Sociales a Gran Escala (Tesis de Maestría). Centro de investigación y de estudios avanzados del Instituto Politécnico Nacional, México. Recuperado de https://www.cs.cinvestav.mx/tesisgraduados/2010/resumenCristianMejia.html

Montes, M., García, A., y Menor, J. (2018). Los vídeos de los adolescentes en YouTube: Características y vulnerabilidades digitales. Comunicar, 24(54), 61-69. Recuperado de https://doi.org/10.3916/C54-201806

Muiños, P. (2017). Los influencers en el Protocolo. Un caso de estudio. Revista Estudios Institucionales, 4(6), 6778. doi: 10.5944/eeii.vol.4.n.6.2017.18826

Ostad, S. A. (2000). Cognitive subtraction in a developmental perspective: Accuracy, speed-of-processing and strategy-use differences in normal and mathematically disabled children. Focus on Learning Problems in Mathematics, 22(2), 18-32. doi: 10.1080/0885625990140103

Pavani, A. M. B., y Temporão, G. P. (2021). M-Learning: Are We Ready to Go Mobile? En M.E. Auer, y T. Tsiatsos (eds.), Advances in Intelligent Systems and Computing: Vol. 1192. Internet of Things, Infrastructures and Mobile Applications (pp. 317-326). Springer, Cham. doi: 10.1007/978-3-030-49932-7_31

Pérez, A., Castro, A., y Fandos, M. (2018). La competencia digital de la Generación Z: claves para su introducción curricular en la Educación Primaria. Comunicar, 24(49), 71-80. doi: 10.3916/C49-2016-07

Pérez, M. (2016). Influencer engagement, una estrategia de comunicación que conecta con la generación millennial (Trabajo de Fin de Grado). Universidad de Alicante, Alicante. Recuperado de https://core.ac.uk/display/43566971

Pérez-Sanagustín, M., Alario-Hoyos, C. y Kloos, C. D. (2014). FLINN: A framework to characterize technology enhanced formal, non-formal and informal learning situations. International Journal of Human Capital and Information Technology Professionals, 5(2), 38-51. doi: 10.4018/ijhcitp.2014040104

Ponce, I. (2012). Redes Sociales. Observatorio Tecnológico: Ministerio de Educación, Cultura y Deporte Gobierno de España. Recuperado de http://recursostic.educacion.es/observatorio/web/ca/internet/web20/1043-redes-sociales?showall=1

Rodríguez, M. C., y Fernández, J. (2017). Uso del recurso de contenido en el aprendizaje en línea: YouTube. Apertura: Revista de Innovación Educativa, 9(1), 22-31. doi: 10.18381/Ap.v9n1.1018

Romeu-Fontanillas, T., Guitert-Catasús, M., Raffaghelli, J. E., y Sangrà, A. (2020). Mirroring learning ecologies of outstanding teachers to integrate ICTs in the classroom. Comunicar, 28(62), 31-42. doi: 10.3916/C62-202003 
Ruiz, F. J. (2019). Los" influencers" en la comunicación local. En C. Gonzalez, y C. Fanjul (eds.), COMLOC 2018: el negocio publicitario en la sociedad digital (pp. 121-140). España: Universitat Jaume I. Recuperado de https://dialnet.unirioja.es/servlet/articulo?codigo=6945645

Scolari, C. (2018). Adolescentes, medios de comunicación y culturas colaborativas. Aprovechando las competencias transmedia de los jóvenes en el aula. Barcelona: Ce.Ge.

Scolari, C. A., Masanet, M.J., Guerrero-Pico, M., y Establés, M. J. (2018). Transmedia literacy in the new media ecology: Teens' transmedia skills and informal learning strategies. El Profesional de la Información, 27(4), 801-812. doi: 10.3145/epi.2018.jul.09

Siegenthaler, R., Miranda, A., Mercader, J., y Presentación, M. J. (2017). Habilidades matemáticas iniciales y dificultades matemáticas persistentes. International Journal of Developmental and Educational Psychology. Revista INFAD de Psicología, 3(1), 233-242. doi: 10.17060/ijodaep.2017.n1.v3.992

Tabuenca, B., Ternier, S., y Specht, M. (2013). Patrones cotidianos en estudiantes de formación continua para la creación de ecologías de aprendizaje. Revista de Educación a Distancia, (37). Recuperado de https://dialnet.unirioja.es/servlet/articulo?codigo=4955092

Torres, K., Álvarez, L., Gutiérrez, B., y Ávila, J.H. (2012). Análisis de Redes Sociales en el contexto virtual. En J.H. Ávila (ed.), Redes sociales y Análisis de Redes: aplicaciones en el contexto comunitario y virtual (pp.168201). Colombia: Corporación Universitaria Reformada. Recuperado de https://dialnet.unirioja.es/servlet/articulo?codigo=4131776

Unal, S., y Ozturk, I. H. (2012). Barriers to ITC integration into teachers' classroom practices: Lessons from a case study on social studies teachers in Turkey. World Applied Sciences Journal, 18(7), 939-944. doi: 10.5829/idosi.wasj.2012.18.07.1243

Wasserman, N. H., Quint, C., Norris, S. A., y Carr, T. (2017). Exploring flipped classroom instruction in Calculus III. International Journal of Science and Mathematics Education, 15(3), 545-568. doi: 10.1007/s10763-0159704-8

Wastiau, P., Blamire, R., Kearney, C., Quittre, V., Van de Gaer, E., y Monseur, C. (2013). The Use of ICT in Education: a survey of schools in Europe. European Journal of Education, 48(1), 11-27. doi:

10.1111/ejed.12020

Wei, J., y Meng, F. (2021). How opinion distortion appears in super-influencer dominated social network. Future Generation Computer Systems, 115, 542-552. doi: 10.1016/j.future.2020.09.011

Esta obra está bajo una Licencia Creative Commons Attribución-NoCommercial 4.0 International

(cc) BY-NC 